\title{
Fibrossarcoma uretral primário em cadela
}

\author{
[Primary urethral fibrosarcoma in a female dog] \\ M.M.V. Silva ${ }^{1}$, L.Z. Crivelenti ${ }^{2}$, C. Momo ${ }^{3}$, D.K. Honsho ${ }^{3}$ \\ ${ }^{1}$ Médica veterinária do setor de anestesiologia do Hospital Veterinário Metodista \\ ${ }^{2}$ Aluno de pós-graduação - Universidade Estadual de São Paulo - UNESP \\ Campus de Jaboticabal - Av. Pedro Marquês, 235 - Jd Universitário \\ 14882-222 - Jaboticabal, SP \\ ${ }^{3}$ Universidade de Franca - Franca, SP
}

\begin{abstract}
RESUMO
Relatou-se um caso de fibrossarcoma localizado na uretra média de uma cadela, sem raça definida, que apresentava sinais de polaquiúria, estrangúria e hematúria no início da micção. $\mathrm{O}$ animal também apresentava poliúria, polidipsia, êmese e anorexia há dois dias. Os exames complementares levaram ao diagnóstico de hidronefrose bilateral, hipoplasia renal direita e massa em uretra causando dilatação dorsal de sua porção média. A biópsia não foi realizada antemortem devido ao estado geral do paciente, que, mesmo com a instituição de tratamento para doença renal crônica, não apresentou progresso. A uretrocistografia retrógrada e a histologia foram os exames decisivos para detectar o fibrossarcoma uretral. A ultrassonografia e a citologia por sondagem traumática não foram conclusivos, devido ao fato de a massa apresentar-se em região media de uretra e o caráter pouco esfoliativo da neoplasia.
\end{abstract}

Palavras-chave: cadela fibrossarcoma, uretra e uretrocistografia

\begin{abstract}
A case of fibrosarcoma located at medium portion of the urethra of a female dog which presented pollakiuria, estranguria and hematuria at the beginning of urination was reported. The patient also presented polyuria, polydipsia, emesis and anorexia for two days. The complementary exams lead to the diagnosis of bilateral hydronephrosis, right renal hypoplasy and a mass in the urethra causing a dorsal dilatation at its medium portion. A biopsy was not performed antemortem because the general condition of the patient, which even with the institution of treatment for chronic kidney disease, showed no progress. The retrograde cystourethrography and histopathology examinations were decisive to detect urethral fibrosarcoma. Ultrasonography and cytology through traumatic probe were inconclusive, due to the mass location and its exfoliating properties.
\end{abstract}

Keywords: bitch, fibrosarcoma, urethra and cystourethrography

\section{INTRODUÇÃO}

Os tumores de uretra são raros em cães (Smeak, 2008), sendo os sinais clínicos disúria, hematúria e estrangúria os principais motivos da consulta inicial (Carlton, 2002; Meuten, 2002). Porém, alguns cães podem ainda apresentar constipação, tenesmo uretrite e noctúria como manifestações do quadro (Smeak, 2008). As neoplasias uretrais acometem mais frequentemente animais idosos e fêmeas. Aparentemente não há predileção por

Recebido em 10 de março de 2011

Aceito em 8 de setembro de 2011

E-mail: crivelenti_lz@yahoo.com.br raça, embora alguns autores acreditem ocorrer uma maior predisposição na raça Beagle (Tarvin et al., 1978; Wilson et al., 1979; Ryan e Holshuh, 1981; Stone, 1985; Norris et al., 1992; Meuten, 2002;). As principais neoplasias encontradas no sistema urogenital são os carcinomas uroteliais, fibromas, leiomiossarcomas e leiomiomas (Zinkl, 2009).

O diagnóstico de neoplasias uretrais pode ser dificultoso devido ao local e à possibilidade de o animal permanecer sem sinais clínicos evidentes 
(Norris et al., 1992; Meuten, 2002). Sendo assim, é importante que se esgotem os recursos pouco invasivos, que vão desde o toque retal (White et al., 1996) ao raio-X contrastado, citologia esfoliativa do canal da uretral, citologia aspirativa por agulha fina guiada por ultrassom e biópsia por laparotomia (Nyland et al., 2002).

Os fibrossarcomas são neoplasias malignas mesenquimais de fibroblastos que produzem tecido conjuntivo e colágeno localizados principalmente na pele, tecido subcutâneo e cavidade oral. Caracterizam-se como neoplasia altamente infiltrativa e recidivante; e apesar de incomum, podem fazer metástases em linfonodos, pulmões e vértebras, sendo os dois últimos locais de menor ocorrência (Carlton, 2002; Meuten, 2002; Gomes, 2003). Macroscopicamente são tumores brancoacinzentados, com crescimento variável. Histologicamente as células podem se apresentar desde entrelaçadas, altamente indiferenciadas ou até fusiformes. Possuem núcleos de redondos a ovoides, frequentemente em mitose, até células alongadas entremeadas com feixes que se assemelham ao tecido conjuntivo imaturo. Ademais, pleomorfismo, hipercromasia e células multinucleadas são achados comuns ao fibrossarcoma (Carlton, 2002).

A exérese de massas uretrais geralmente é complexa, sendo a uretroplastia o método de escolha de reparação/reconstituição da uretra. Nestes casos a retirada com margem de segurança é importante e, para isso, é necessária a utilização de enxertos autólogos, como o uso de fascia lata (Atalan et al., 2005), flaps vaginais (White et al., 1996; Blaivas e Heritz, 1996) ou mucosa bucal (Silva et al., 2002). Xenoenxertos utilizando submucosa intestinal suína mostrou-se um bom recurso para extensas cirurgias reparativas da uretra (Palma et al., 2003).

O presente trabalho relata um caso de uma cadela que apresentava doença renal crônica e hidronefrose bilateral decorrentes de fibrossarcoma uretral, causando obstrução parcial da uretra média.

\section{CASUÍSTICA}

Uma cadela, sem raça definida, de 16 anos, $8,6 \mathrm{~kg}$, foi atendida em Hospital Veterinário escola, por apresentar polaquiúria, estrangúria e hematúria no início da micção, e também apresentava poliúria, polidipsia, êmese e anorexia há dois dias. Ao exame físico, o animal apresentou-se, apenas, com moderada desidratação. Laboratorialmente foram avaliados hemograma, exames bioquímicos e urinálise, os quais evidenciaram alterações compatíveis com quadro de DRC (Tab. 1 e 2).

O ultrassom revelou grau leve de hidronefrose bilateral e hipoplasia renal direita, sem alterações visíveis na bexiga e na uretra. O comprometimento uretral foi visibilizado com o emprego da uretrocistografia retrógrada com duplo contraste, na qual foi encontrada uma massa causando dilatação dorsal da uretra da porção média (Fig. 1).

Tabela 1. Hemograma e bioquímica sérica ao primeiro dia de uma cadela, sem raça definida, com doença renal crônica e hidronefrose

\begin{tabular}{lcc}
\hline & Hematologia & \\
\hline Hemácias & 5,7 & $\mathrm{X} 1000000 / \mu \mathrm{l}$ \\
Hematócrito & 44,0 & $\%$ \\
Hemoglobina & 15,1 & $\mathrm{~g} / \mathrm{dl}$ \\
Plaquetas & 227.000 & $/ \mu 1$ \\
\hline & Bioquímica sérica & \\
\hline ALT & 64,1 & $\mathrm{U} / \mathrm{l}$ \\
Fosfatase Alcalina & 124 & $\mathrm{U} / 1$ \\
Ureia & 164,0 & $\mathrm{mg} / \mathrm{dl}$ \\
Creatinina & 3,4 & $\mathrm{mg} / \mathrm{dl}$ \\
Fibrinogênio & 400,0 & $\mathrm{mg} / \mathrm{dl}$ \\
Proteína total & 6,4 & $\mathrm{dl}$ \\
\hline
\end{tabular}


Tabela 2. Dados referentes à urinálise da cadela com doença renal crônica e hifronefrose

\begin{tabular}{ll} 
& \multicolumn{1}{c}{ Urinálise } \\
\hline Cor & Amarelo-claro \\
Odor & Sui generis \\
Aspecto & Ligeiramente turvo \\
Densidade & 1,018 \\
Ph & 6,0 \\
Proteínas & $30 \mathrm{mg} / \mathrm{dl}(+)$ \\
Sangue oculto & $(++)$ \\
Leucócitos & Incontáveis / CGA \\
Células & Raras células epiteliais uretrais e da bexiga urinária \\
Cilindros & Ausentes \\
Cristais & Ausentes \\
Outros & Discreta bacteriúria $(+)$ \\
\hline
\end{tabular}

Após a localização da massa, realizou-se sondagem uretral traumática e leitura de lâmina após a citocentrifugação da amostra. Foram encontradas diversas células normais de escamação de epitélio renal, uretral e vesical evidenciando apenas uma célula em mitose. Recomendou-se a laparotomia para exérese da neoplasia, porém o proprietário optou pelo tratamento conservativo da doença renal crônica, alegando avançada idade do animal e poucas condições financeiras. $\mathrm{O}$ tratamento constituiu-se de fluidoterapia parenteral com solução de Ringer Lactato adicionado de polivitamínico $(90 \mathrm{~mL} / \mathrm{kg} / \mathrm{dia})$ metoclorpramida $(0,3 \mathrm{mg} / \mathrm{kg})$ e ranitidina $(2 \mathrm{mg} / \mathrm{kg})$ a cada 12 horas pela via subcutânea. Para monitoração do quadro, foram realizados exames periódicos de ureia e creatinina (Tab. 3).

Os rins apresentavam contornos irregulares, pelve dilatada e hipoplasia do rim direito. Ao exame histológico, a massa da uretra resultou em fibrossarcoma, a qual apresentou células alongadas, originadas de fibroblastos e fibrócitos com moderado pleomorfismo e raras mitoses, distribuídas em "ninhos" (Fig. 3).

Após 25 dias, mesmo com o tratamento, o animal apresentou piora do quadro, sendo, então, eutanasiado. À necropsia foi evidenciada uma massa firme esbranquiçada de $4 \mathrm{~cm}$ de diâmetro na uretra média (Fig. 2).

\section{DISCUSSÃO}

A citologia aspirativa por sondagem não mostrou ser efetiva para a avaliação deste tipo de neoplasia uretral, o que pode ser explicado pela baixa capacidade esfoliativa do fibrossarcoma (Brandão, et al., 2007).

Os fibrossarcomas são neoplasias raras em cães e gatos, podendo ser encontrados em qualquer tecido, apesar de encontrado com maior facilidade no tecido subcutâneo (Wilson et al., 1979;). Ainda hoje, desconhecem-se relatos de fibrossarcoma uretral. A neoplasia uretral obstruiu o fluxo urinário e, com a cronicidade do caso, causou refluxo da urina para os ureteres e pelve renal, resultando em hidronefrose bilateral. Somado à hipoplasia unilateral renal, os efeitos da neoplasia uretral pioraram a azotemia, do caso em tela.

A azotemia prolongada, associada à alteração da função renal, culmina na síndrome urêmica com o surgimento de diversos sinais (Smeak, 2008), como o desequilíbrio hídrico natrêmico, anemia, intolerância a carboidratos, distúrbios neurológicos, distúrbios gastroentéricos, osteodistrofias, deficiência imunológica, acidose metabólica e processos inflamatórios que predispõem à fibrose e esclerose renal (Pollock, 1968), levando à piora do quadro, consequentemente ao óbito, como o quadro descrito neste relato.

Tabela 3. Valores de ureia e creatinina da cadela com doença renal crônica e hidronefrose no decorrer do tratamento

\begin{tabular}{lccc} 
& $4^{\circ}$ dia & $7^{\circ}$ dia & $9^{\circ}$ dia \\
\hline Ureia & $54,4 \mathrm{mg} / \mathrm{dL}$ & $53,8 \mathrm{mg} / \mathrm{dL}$ & $75,2 \mathrm{mg} / \mathrm{dL}$ \\
Creatinina & $1,9 \mathrm{mg} / \mathrm{dL}$ & $2,2 \mathrm{mg} / \mathrm{dL}$ & $3,1 \mathrm{mg} / \mathrm{dL}$ \\
\hline
\end{tabular}



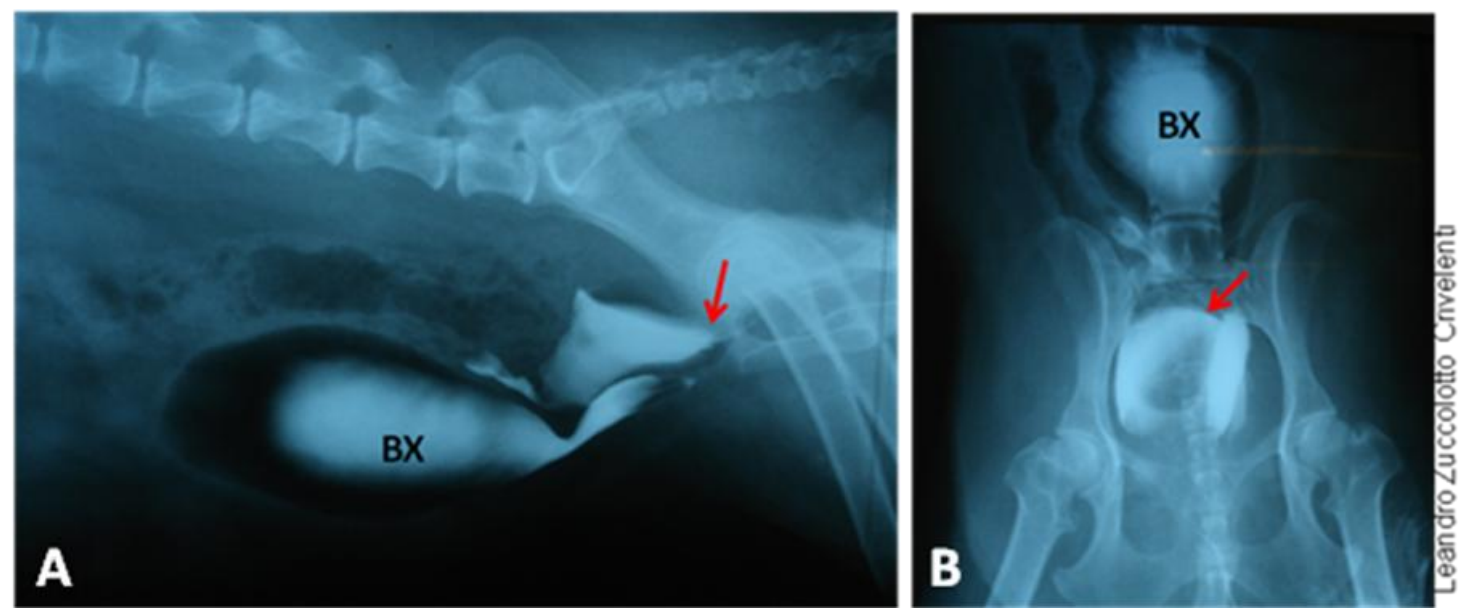

Figura 1. Uretrocistografia retrógrada com duplo contraste em cadela com doença renal crônica e hidronefrose, onde (A) posição laterolateral direita com contraste extravasando na uretra dorsal, e em (B) na posição ventrodorsal evidenciando a massa em região média da uretra. As setas indicam a massa.
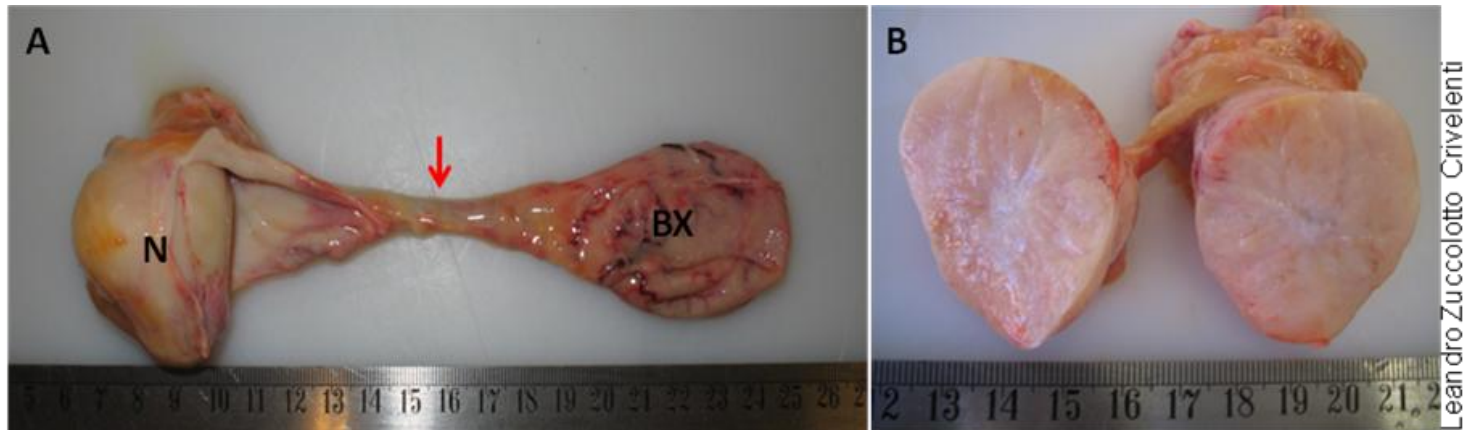

Figura 2. Cadela com doença renal crônica e hidronefrose; em (A) mostrando a neoplasia em região de uretra média, a seta indica o percurso da uretra. (B) $\mathrm{O}$ corte da massa evidenciando esbranquiçada e firme.
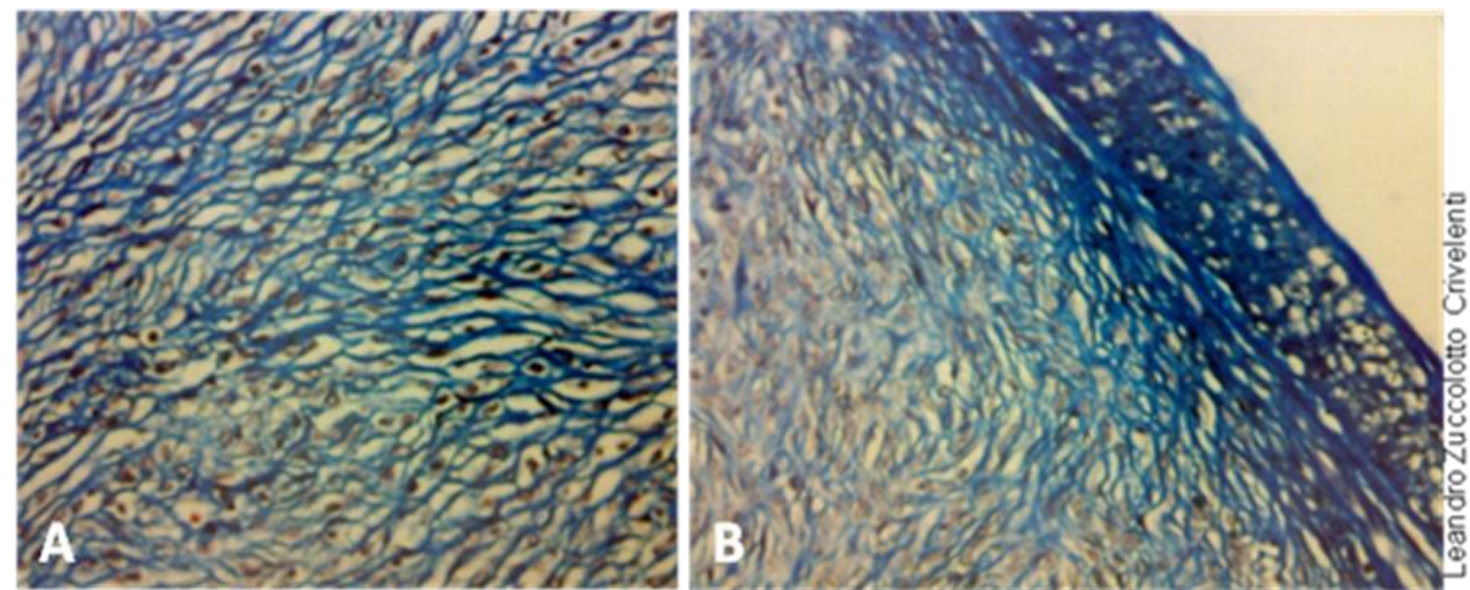

Figura 3. Cadela com doença renal crônica e hidronefrose, onde (A) fibrossarcoma apresentando células alongadas, originadas de fibroblastos e fibrócitos com moderado pleomorfismo, e (B) raras mitoses distribuídas em "ninhos" 
Não foi possível estabelecer uma imagem ultrassonográfica da neoplasia devido ao fato de região uretral média ser asilada por ossos da pube, o que não permite a formação da imagem e impossibilita a realização da punção biópsia aspirativa guiada. A uretrocistografia retrógrada de duplo contraste foi uma opção para a identificação da neoplasia uretral conforme a indicação no diagnóstico das alterações urinárias inferiores (Valli, 1995; Carlton, 2002).

Ao iniciar o tratamento de doença renal crônica, o animal apresentou melhora transitória, porém, após alguns dias, agravou-se o quadro. Isso talvez se deva muito provavelmente ao caráter obstrutivo da neoplasia, que pode ter levado a maior grau de estase renal (hidronefrose), culminando no quadro descompensatório irreversível e na eutanásia da paciente.

O diagnóstico definitivo foi realizado por meio do exame histológico compatível com fibrossarcoma. O tipo de tumor encontrado dificultou o diagnóstico devido à sua baixa característica esfoliativa e localização, impossibilitando o diagnóstico por meio de exames rotineiramente utilizados para neoplasias uretrais. Corroborando com alguns autores, o paciente não apresentou metástase (Carlton, 2002; Pena, et al., 2000; Gomes 2003).

\section{CONCLUSÕES}

As neoplasias uretrais são de difícil detecção devido a sua localização e aos sinais clínicos generalizados. A uretrocistografia e a histotologia foram os exames mais indicados para detectar o fibrossarcoma uretral. A ultrassonografia e a citologia por sondagem traumática não foram conclusivas, devido ao fato de a massa apresentar-se em região média de uretra e ao seu caráter pouco esfoliativo.

\section{REFERÊNCIAS BIBLIOGRÁFICAS}

ATALAN, G.; SOZMEN, M.; OZAYDIN, I. et al. Repair of urethral defects using fascia lata atutografts in dogs. Vet. Surg., v.34, p.514-518, 2005.

BLAIVAS, J.G.; HERITZ, D.M. Vaginal flap reconstruction of the urethra and vesical neck in women: a report of 49 cases. J. Urol., v.155, p.1014-1017, 1996.
BRANDÃO C.V.S. et al. Fibrossarcoma em cães - relato de dois casos. In: CONPAVEPA CONGRESSO PAULISTA DA ANCLIVEPA, 7., 2007, São Paulo. Anais... S.P.: Anclivepa, 2007.

CARLTON, W.W.; MC GAVIN, M.D. Patologia veterinária especial de Thomsom. 2. ed. Porto Alegre: Artmed, 2002, p.742-743.

GOMES, C. Fibrossarcoma multicêntrico em um cão: relato de caso. In: CONGRESSO BRASILEIRO DA ANCLIVEPA. 24., Anais... Belo Horizonte: Editora, v.2, p.78, 2003.

MEUTEN, D.J. Tumors of the urinary system. In: MEUTEN, D.J. Tumors in domestic animals. 4.ed. Ames: Iowa State, 2002. p.509-546.

NORRIS, A.M. et al. Canine bladder an urethral tumors: a retrospective sudy of 115 cases (19801985). J. Vet. Intern. Med., v.6, p.145-153, 1992.

NYLAND, T.G.; WALLACK, S.T.; WISNER, E.R. Needle-tract implantation following usguided fine-needle aspiration biopsy of transitional cell carcinoma of the bladder, urethra, and prostate. Vet. Radiol. Ultrasound., v.43, p.50-53, 2002.

PALMA, P.C.R.; RICCETTO, C.L.Z.; THIEL, M. Emprego da submucosa intestinal suína em uretroplastias complexas. Rev. Col. Bras. Cir., v.30, p.319-321, 2003.

PENA, S.B.; FLORENTINO, K.C.; BATISTA, J.C. et al. Fibrossarcoma canino: relato de caso. In: SIMPÓSIO DE PATOLOGIA VETERINÁRIA, 2., 2000, Garça. Anais... Garça: SEPAVET, 2000.

POLLOCK, S. Urethral carcinoma in the dog: a case report. J. Am. Vet. Radiol. Soc., v.9, p.9598, 1968 .

RYAN, C.P.; HOLSHUH, H.J. Urethral adenocarcinoma in a dog. Vet. Med. Small. Anim. Clin., v.76, p.1315-1317, 1981.

SILVA, F.F.; PAULO, N.M.; BRITO, G.A. et al. Avaliação da triangulação da anastomose término-terminal de fragmento de mucosa bucal na reconstrução uretral: estudo experimental no cão. Acta Cir. Bras., v.17, p.327-331, 2002.

SMEAK, D. In: BRICHARD, S.J.; SHERDING, R.G. Manual Saunders clínica de pequenos animais. 3.ed. São Paulo, SP: Roca, 2008. 
STONE, E.A. Urogenital tumors. Vet. Clin. North. Am. Small. Anim. Pract., v.15, p.597-608, 1985.

TARVIN, G. et al. Primary urethral tumors in dogs. J. Am. Vet. Med. Assoc., v.172, p.931-933, 1978 .

VALLI, V.E. et al. Pathology of canine bladder and urethral cancer and correlation with tumour progression and survival. J. Comp. Path., v.113, p.113-130, 1995.
WILSON, G.P. et al. Canine urethral cancer. J. Am. Anim. Hosp. Assoc., v.15, p.741-744, 1979.

WHITE, R.N.; DAVIES, J.V.; GREGORY, S.P. Vaginourethroplasty for treatment of urethral obstruction in the bitch. Vet. Surg., v.25, p.503510, 1996

ZINKL, J.G. Exame do sedimento urinário. In: COWELL, R.L.; TYLER, R.D.; MEINKOTH, J.H.; DENICOLA, D.B. Diagnóstico citológico e hematológico de cães e gatos. 3.ed. São Paulo: MedVet, 2009, p.350-368. 